Article

\title{
Energy Analysis of Highway Electric HDV Platooning Considering Adaptive Downhill Coasting Speed
}

\author{
Miaomiao Hu ${ }^{1, *}$ and Peter Bauer ${ }^{2, *}$ \\ 1 Department of Electrical and Computer Engineering, University of Florida, Gainesville, FL 32611, USA \\ 2 Department of Electrical Engineering, University of Notre Dame, Notre Dame, IN 46556, USA \\ * Correspondence: mhu1@ufl.edu (M.H.); pbauer@nd.edu (P.B.)
}

check for

updates

Citation: Hu, M.; Bauer, P. Energy Analysis of Highway Electric HDV Platooning Considering Adaptive Downhill Coasting Speed. World Electr. Veh. J. 2021, 12, 180. https:// doi.org/10.3390/wevj12040180

Academic Editor: Ching-Ming Lai

Received: 8 September 2021

Accepted: 30 September 2021

Published: 7 October 2021

Publisher's Note: MDPI stays neutral with regard to jurisdictional claims in published maps and institutional affiliations.

Copyright: (c) 2021 by the authors. Licensee MDPI, Basel, Switzerland. This article is an open access article distributed under the terms and conditions of the Creative Commons Attribution (CC BY) license (https:// creativecommons.org/licenses/by/ $4.0 /)$.

\begin{abstract}
Increase of non-renewable energy consumption and $\mathrm{CO}_{2}$ emissions has brought heavy burdens to our planet. Heavy-duty vehicles as a large energy consumer benefit a lot from platooning due to reduced air drag. Comparing to ICE trucks, electric trucks can gain more energy savings from platooning while also reducing $\mathrm{CO}_{2}$ emissions. This paper explores the energy consumption of electric HDV platooning under highway situations and proposes an adaptive downhill coasting speed method with regenerative braking. Simulations show that electric HDV platooning can reach at most $33.4 \%$ energy savings with our proposed adaptive coasting speed profile. With a sacrifice of $22.2 \%$ travel time, the energy savings can be further increased to $49.3 \%$.
\end{abstract}

Keywords: electric trucks; heavy-duty; fleet; downhill coasting; regenerative braking

\section{Introduction}

Due to the increasing number of vehicles all over the world, the amount of nonrenewable energy consumption and $\mathrm{CO}_{2}$ emissions has increased drastically, which has brought heavy burdens to our planet. According to the report [1], although medium-duty vehicles (MDVs) and heavy-duty vehicles (HDVs) only account for $4 \%$ of all operating vehicles, they consume almost $20 \%$ of the transportation energy. The HDV industry is even more sensitive to fuel consumption mainly due to the large vehicle mass, long operating range and heavy loads [2]. Considering the energy consumption of HDVs, especially for highway applications, air drag is an important factor to be considered. In literature [3], the air drag constitutes approximately $23 \%$ of the total energy consumptions of a 40 ton truck under average road and driving conditions. It is necessary to establish a good control strategy to realize the reduction of energy consumption for HDV users.

The topic of highway platooning of conventional HDVs has been the subject of intense research over the years due to its potential for reducing air drag and improving fuel efficiency. Researchers from Auburn University and Peloton Technology proposed a prototype of driver-assistive truck platooning (DATP) and showed that the platooning system has significant positive safety and fuel savings potential for heavy truck operations [4]. Daimler Trucks implemented a truck platoon with three identical Mercedes-Benz Actros trucks and participated in the European Truck Platooning challenge [5]. Volvo leads the SARTRE project and developed a platoon of one truck and four cars with a relative distance of $4 \mathrm{~m}$ between vehicles [6]. The work in [7,8] show the fuel consumption reduction for ICE trucks via platooning is between $4.7 \%$ and $7.7 \%$ depending on speed, vehicle type, vehicle mass and relative distance. A DATP system was tested in $[9,10]$ and demonstrated the relationship between energy savings and relative distances. With a shorter relative distance, researchers in [11] proved $14.2 \%$ of energy savings in a three-truck platoon.

Platooning is a good way to help reduce the air drag, however, control of HDVs in a platoon remains a challenging problem in the real world. Due to large mass, heavy loads and limited engine power, maintaining a certain distance in the platoon is very hard for HDV users, especially when including different road grades [12]. Given the information of 
road topology, look-ahead control (LAC) [13] has been a popular method to deal with this problem. Given the information of road grades, the LAC method calculates the optimal speed profile for vehicles within a certain time interval. The model predictive control (MPD) method was used in [14] to obtain an optimal speed profile via dynamic programming. A cooperative look-ahead controller for platooning (CLAC) was proposed in [7] to reduce the fuel consumption of HDV platoons. This work considers the HDVs in a platoon to be a group and changes their speeds at certain road points together instead of maintaining a specific distance for each individual. They achieved a $14 \%$ energy reduction in a downhill segment. The work in [15] shows a connected cruise control method for HDVs considering motion information and vehicle to vehicle (V2V) communication without assuming carfollowing models for preceding vehicles. Around 10\% fuel economy improvement was proved in their work. A predictive kinetic energy management (PKEM) scheme in [16] was conducted for large electric vehicles considering upcoming environment conditions and traffic state. They computed driver-assistive and corrective outputs in real time based on radar information and prove a $10-12 \%$ energy savings.

While platooning of ICE trucks provides a significant efficiency increase, platooning using electric drives has even greater potential for efficiency improvement. Considering the motor efficiency characteristics of an ICE vehicle, it has a very pronounced area in the efficiency map where thermal conversion efficiency is maximum (or the Brake-specific fuel consumption (BSFC) is minimum). Therefore, for an ICE vehicle, a reduction in air drag leads to a reduction in the required motor torque, which also reduces energy efficiency. This is because the operating point is moved towards lower torques in the torque versus speed map, thus reducing power and efficiency. In essence, the reduction in required power is partially masked by a reduction in efficiency. However, an electric drive system has a wide plateau of almost constant efficiency and therefore the effect of reducing air drag is much less pronounced in electric drives. Hence, electric platoons will have larger efficiency gains than conventional ICE platoons, for all but the lead vehicle. In addition, reducing energy consumption in electric drives in general has many secondary desirable effects: reduced time at charging stations, reduced carbon emissions at the power plant and an extended range. However, very little research was conducted based on electric HDV platooning [17]. Our paper aims to fill this gap through investigating the energy consumptions of highway electric HDV platooning.

Downhill coasting can be used to increase the energy savings for an HDV platoon. During the coasting period, HDVs can cut off the fuel and benefit from the kinetic energy stored in the vehicle. However, the coasting speeds for conventional HDVs in most cases cannot satisfy highway speed requirements. However, with the reduction of air drag in a platoon of electric HDVs, coasting speeds can be realized that fall in the range of acceptable highway speeds. This facilitates the implementation of downhill coasting speeds that adapt to different road grades in order to save energy. In this work, we present an adaptive downhill coasting speed control method with regenerative braking for electric HDV platooning under highway situations. This speed control method allows the electric HDV platoon to adaptively change coasting speeds based on different road grades and highway speed limits, which can help the platoon to realize more energy savings.

The remainder of this paper is organized as follows: The simulation model of road grades, air drag, and coasting speeds are presented in Section 2. The rules of realizing acceptable coasting speeds are explained in Section 3. Finally, the simulation results and conclusions are presented in Sections 4 and 5, respectively.

\section{Model and Assumptions}

\subsection{Profile of Road Grades}

In order to decide if it is realistic to implement coasting during highway travel, typical values of road grades are approximately calculated by using the elevation contour map of most cities and highways in the US [18]. Road grades are roughly $2-4 \%$ in the mountain areas of the US, while in other areas, road grades are mostly less than $2 \%$. Therefore, the 
range $-3 \%$ to $3 \%$ is used as typical values of highway grades in our simulations. Positive values represent uphill while negative values represent downhill.

\subsection{Reduction of Air Drag}

Platooning reduces the air drag coefficient of vehicles within a platoon depending on their position, speed, following distance, etc. [19]. To implement coasting in a platoon, all peer vehicles should have a similar ratio of mass and air drag coefficient so that the coasting speeds will be approximately constant [20]. Hence, only homogenous platoons are considered in this paper.

Relative distance is an important factor when considering the reduction of air drag coefficient in a platoon. According to Alam and Assad's work [6], there will be more reduction in the air drag coefficient with less relative distance between vehicles. More specifically, they conducted the experiments in a platoon of three identical HDVs with an approximate average speed of $70 \mathrm{mph}$. Results show that the air drag coefficient of the second and third vehicle can be reduced by more than $40 \%$ when the relative distance is around 15 meters. The reduction of air drag coefficient can reach at most $70 \%$ with a very small relative distance. Here in our paper, considering the application of highway HDV platooning, it is necessary to maintain certain gaps for safety, so we assume the relative distance is in the range of 15 meters and the air drag reduction is $40 \%$ for all vehicles in the platoon except for the lead vehicle.

\subsection{Energy Model}

Figure 1 shows the longitudinal forces balance on an HDV in motion. Denoting vehicle mass, vehicle speed, rolling resistance coefficient, air drag coefficient, front cross-sectional area, air density and road grade as $m, v, f_{r}, C_{d}, A, \rho$ and $\alpha$ respectively, the power balance at the wheel can be expressed as:

$$
P_{\text {wheel }}=m a v+\frac{1}{2} C_{d} A \rho v^{3}+m g v f_{r} \cos \alpha+m g v \sin \alpha
$$

where $P_{\text {wheel }}$ and $a$ denote the power at the wheel and vehicle acceleration respectively. An incline is associated with a positive $\alpha$, while a decline is associated with a negative $\alpha$.

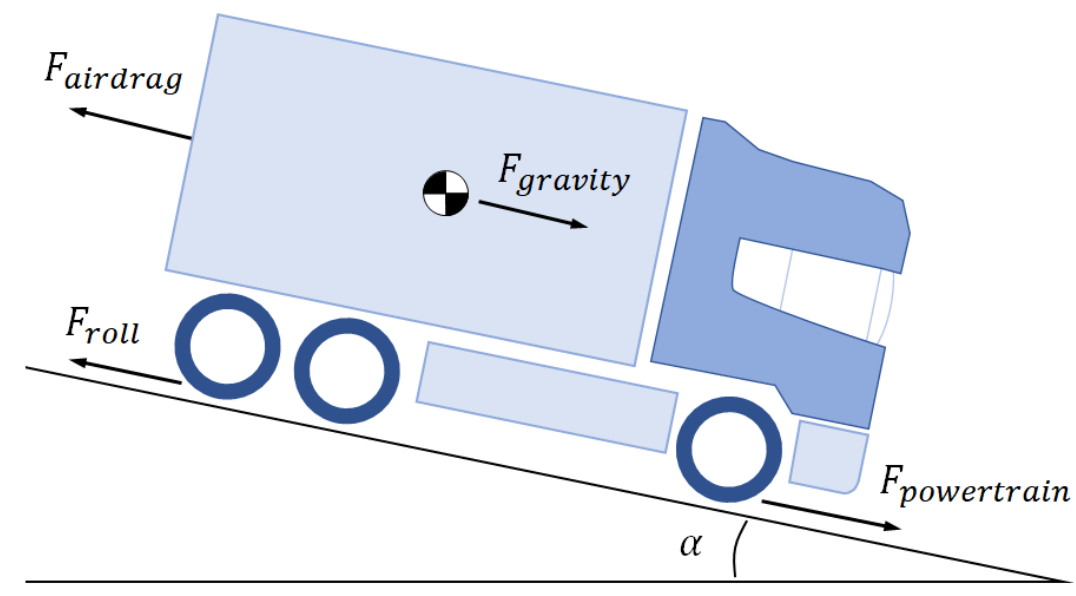

Figure 1. The longitudinal forces for an electric truck in motion.

The energy consumption on a distance segment $\Delta x$ can be represented under steady state conditions as:

$$
E \eta=\frac{1}{2} C_{d} A \rho v^{2} \Delta x+m g f_{r} \cos \alpha \Delta x+m g \sin \alpha \Delta x
$$


where $\Delta x$ is the travel distance segment. The constant forward power flow efficiency is denoted by $\eta$. $E$ denotes the energy at the source, i.e., the battery.

\section{Coasting Speeds}

The coasting speed for conventional HDVs cannot satisfy the requirements of highway speeds in most cases, but the reduction of air drag increases the coasting speed of platoon vehicles to values that are often within the range of acceptable highway speeds.

With $P_{\text {wheel }}=0$ and acceleration $a=0$ during coasting, Equation (1) can be simplified for steady state conditions:

$$
\frac{1}{2} C_{d} A \rho v^{3}+m g v f_{r} \cos \alpha+m g v \sin \alpha=0
$$

The steady coasting speed is then given by

$$
v_{c}=\sqrt{\frac{-2 m g\left(\sin \alpha+f_{r} \cos \alpha\right)}{C_{d} A \rho}} \text { for } f_{r}<\tan (-\alpha), \alpha<0
$$

Typical coasting speeds based on different ratios of $m / C_{d} A$ are shown in Table 1 . According to the acceptable limits of highway speeds ( $50 \mathrm{mph}-100 \mathrm{mph}$ ), we used four types of trucks with different masses to form platoons and calculated the coasting speeds respectively.

Table 1. Coasting speeds (mph) with respect to different declines and ratios of $m / C_{d} A$ for the second truck in a platoon.

\begin{tabular}{cccc}
\hline$m / C_{\boldsymbol{d}} \boldsymbol{A}\left(\mathbf{k g} / \mathbf{m}^{\mathbf{2}}\right)$ & $\boldsymbol{\alpha}=\mathbf{- 1} \%$ & $\alpha=-\mathbf{2} \%$ & $\alpha=-\mathbf{3} \%$ \\
\hline 1000 & 15.1 & 31.5 & 41.8 \\
2000 & 21.4 & 44.5 & $\mathbf{5 9 . 2}$ \\
3000 & 26.2 & $\mathbf{5 4 . 5}$ & $\mathbf{7 2 . 5}$ \\
6000 & 37.0 & $\mathbf{7 7 . 0}$ & 102.5 \\
\hline
\end{tabular}

\section{Simulations}

\subsection{Settings and Assumptions}

In the simulations, all trucks in a platoon are assumed to be identical to each other and the gap between two trucks is $10 \mathrm{~m}$, which causes approximately a $40 \%$ reduction of air drag according to [6]. The energy consumption is considered with/without regenerative breaking gain and a constant powertrain efficiency is assumed to be $80 \%$. Ignoring the effect of wind, temperature and other factors, the gap between two trucks in a platoon is assumed to be constant in each segment. Table 2 shows the parameters of trucks used in our simulations and Table 3 shows the coasting speeds of e-platoons with different $m / C_{d} A$ ratios. Adapted coasting speeds in the platoon are implemented when the declines are $-2 \%$ and $-3 \%$.

Table 2. Electric truck parameters.

\begin{tabular}{cc}
\hline Parameters & Values \\
\hline$m$ & $12,000 / 20,000 / 25,000 / 30,000 \mathrm{~kg}$ \\
$C_{d}$ & 0.8 \\
$A$ & $10 \mathrm{~m}^{2}$ \\
$\rho$ & $1.29 \mathrm{~kg} / \mathrm{m}^{3}$ \\
$g$ & $9.81 \mathrm{~m} / \mathrm{s}^{2}$ \\
$f_{r}$ & 0.007 \\
\hline
\end{tabular}


Table 3. Coasting speeds (mph) of trucks with different $m / C_{d} A$ in a platoon.

\begin{tabular}{ccccc}
\hline Mass $(\mathbf{k g})$ & $m / C_{\boldsymbol{d}} \boldsymbol{A}\left(\mathbf{k g} / \mathbf{m}^{\mathbf{2}}\right)$ & $\alpha=-\mathbf{1} \%$ & $\alpha=-\mathbf{2} \%$ & $\alpha=-\mathbf{3} \%$ \\
\hline 12,000 & 2500 & 23.9 & 49.7 & $\mathbf{6 6 . 2}$ \\
20,000 & 4167 & 30.8 & $\mathbf{6 4 . 2}$ & $\mathbf{8 5 . 5}$ \\
25,000 & 5208 & 34.5 & $\mathbf{7 1 . 8}$ & $\mathbf{9 5 . 5}$ \\
30,000 & 6250 & 37.8 & $\mathbf{7 8 . 7}$ & $\mathbf{1 0 4 . 7}$ \\
\hline
\end{tabular}

Figure 2 shows the profile of road segments with different grades in our simulations. The percentage represents different road grades where negative numbers represent declines and positive numbers represent inclines. The same road profile is used for all trucks with parameters in Table 2 and the total travel time for all cases is approximately constant.

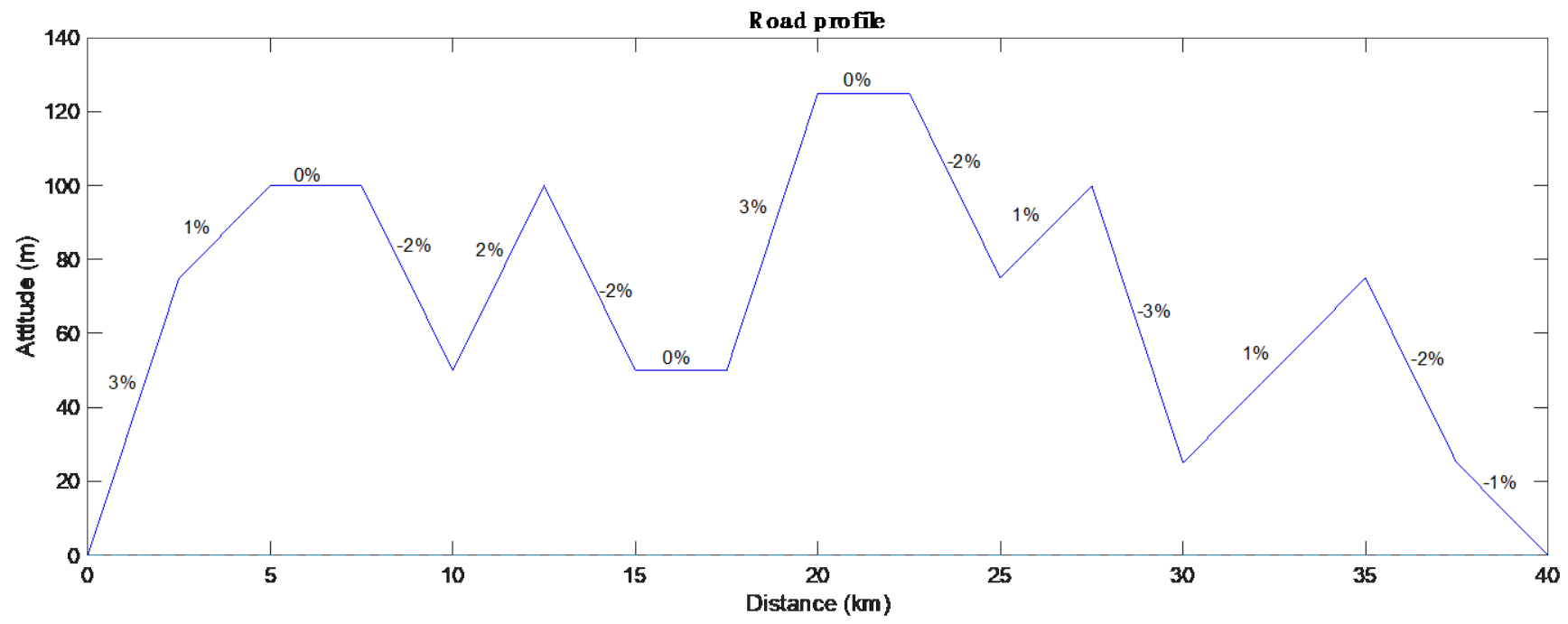

Figure 2. Profile of road segments with different grades.

\subsection{Energy Consumption without Regenerative Braking}

In this part, the energy gains from regenerative braking are ignored and the energy balance is considered for three cases: (a) a single vehicle with a constant speed; (b) a platoon with a constant speed; (c) a platoon with adapted coasting speeds. Table 4 shows the speeds used in different road segments for the three cases and the relative energy savings of case (b) and (c) compared to case (a). Figure 3 shows the energy consumptions at the battery for four different types of trucks.

Table 4. Speeds (mph) and relative energy savings of four types of trucks in three cases without regenerative braking.

\begin{tabular}{ccccccc}
\hline $\begin{array}{c}\text { Mass } \\
(\mathbf{k g})\end{array}$ & $\begin{array}{c}\text { Constant Speed in } \\
\text { Case (a)(b) }\end{array}$ & $\boldsymbol{\alpha} \geq-\mathbf{- 1} \%$ & $\begin{array}{c}\text { Speeds in Case (c) } \\
\boldsymbol{\alpha}=-\mathbf{2} \%\end{array}$ & $\boldsymbol{\alpha = - 3 \%}$ & \multicolumn{2}{c}{ Energy Savings } \\
Case (b)
\end{tabular}

From Table 4, relative energy savings of a platoon with a constant speed in case (b) can reach $32 \%$ compared to a single truck with a constant speed in case (a). Gains for heavier trucks are even higher and can reach $32.8 \%$. For platoons with adapted speeds in case (c), relative energy savings are around $33 \%$ and depend slightly on truck mass. These numbers are significantly higher than in ICE platoons. 

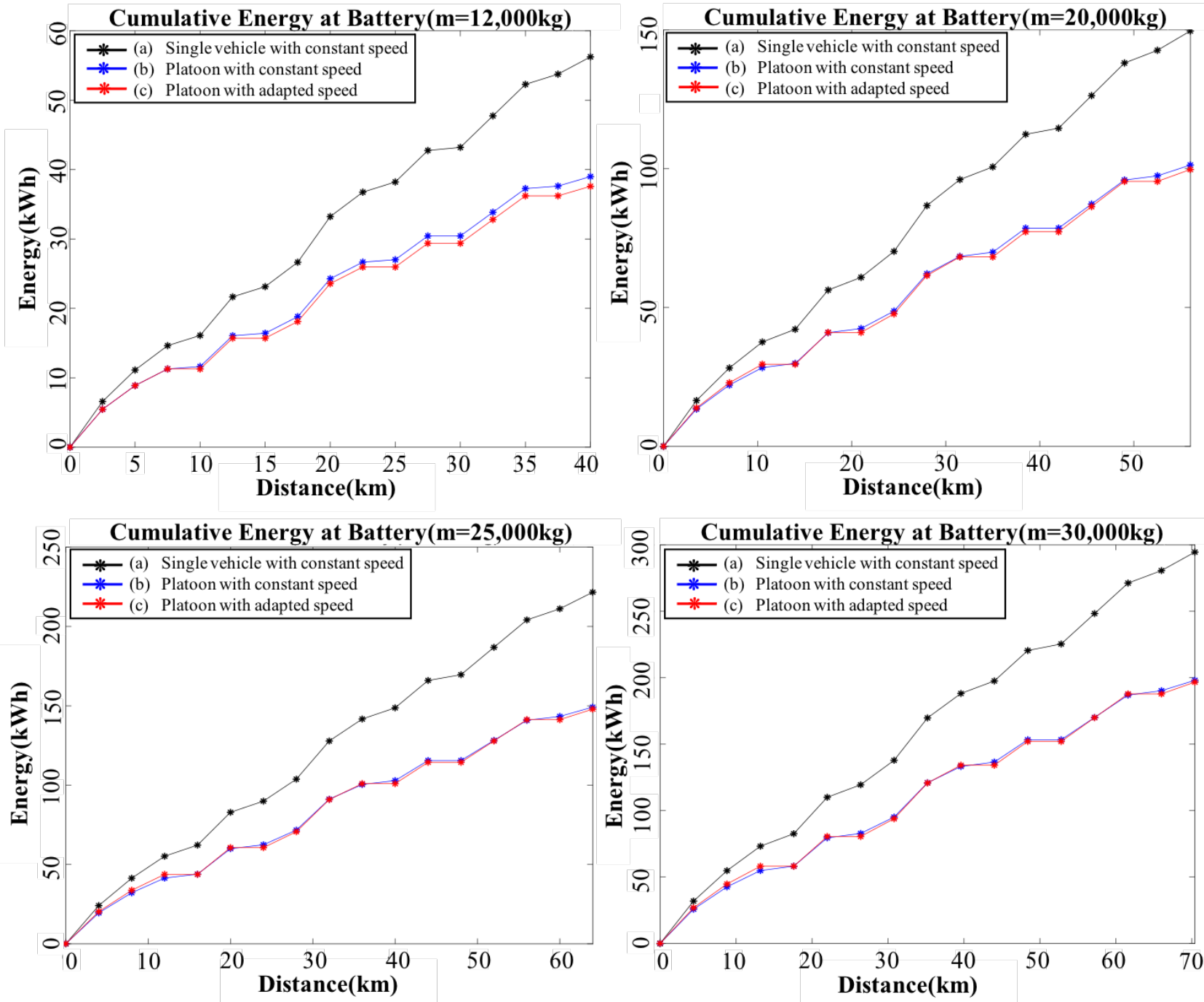

Figure 3. Energy consumptions of four types of trucks with different $m / C_{d} A$.

\subsection{Energy Consumption with Regenerative Braking}

In this part, the energy balance is simulated with regenerative breaking in three cases: (a) a single vehicle with a constant speed; (b) a platoon with a constant speed; (c) a platoon with adapted coasting speeds.

For case (a) and (b), trucks brake to the constant speed to obtain energy savings. For case (c), to get larger regenerative efficiency, trucks brake to the lowest acceptable highway speed limit (50 mph). The regenerative efficiency is assumed to be $25 \%$ for $12,000 \mathrm{~kg}$ trucks and $50 \%$ for $20,000 \mathrm{~kg}, 25,000 \mathrm{~kg}$ and $30,000 \mathrm{~kg}$ trucks. Table 5 shows the speeds used in different road segments for the three cases and the relative energy savings of cases (b) and (c) compared to the case (a). Figure 4 shows the energy consumptions at the battery for four types of trucks.

Table 5. Speeds (mph) and relative energy savings of four types of trucks in three cases with regenerative braking.

\begin{tabular}{|c|c|c|c|c|c|c|}
\hline \multirow{2}{*}{$\begin{array}{c}\text { Mass } \\
\text { (kg) }\end{array}$} & \multirow{2}{*}{$\begin{array}{l}\text { Constant Speed in } \\
\text { Case }(a)(b)\end{array}$} & \multicolumn{3}{|c|}{ Speeds in Case (c) } & \multicolumn{2}{|c|}{ Energy Savings } \\
\hline & & $\alpha \geq-1 \%$ & $\alpha=-2 \%$ & $\alpha=-3 \%$ & Case (b) & Case (c) \\
\hline 12,000 & 55.9 & 57.5 & 50.0 & 50.0 & $31.1 \%$ & $32.1 \%$ \\
\hline 20,000 & 55.9 & 57.5 & 50.0 & 50.0 & $27.9 \%$ & $28.7 \%$ \\
\hline 25,000 & 55.9 & 57.5 & 50.0 & 50.0 & $27.1 \%$ & $28.2 \%$ \\
\hline 30,000 & 55.9 & 57.5 & 50.0 & 50.0 & $27.7 \%$ & $29.0 \%$ \\
\hline
\end{tabular}



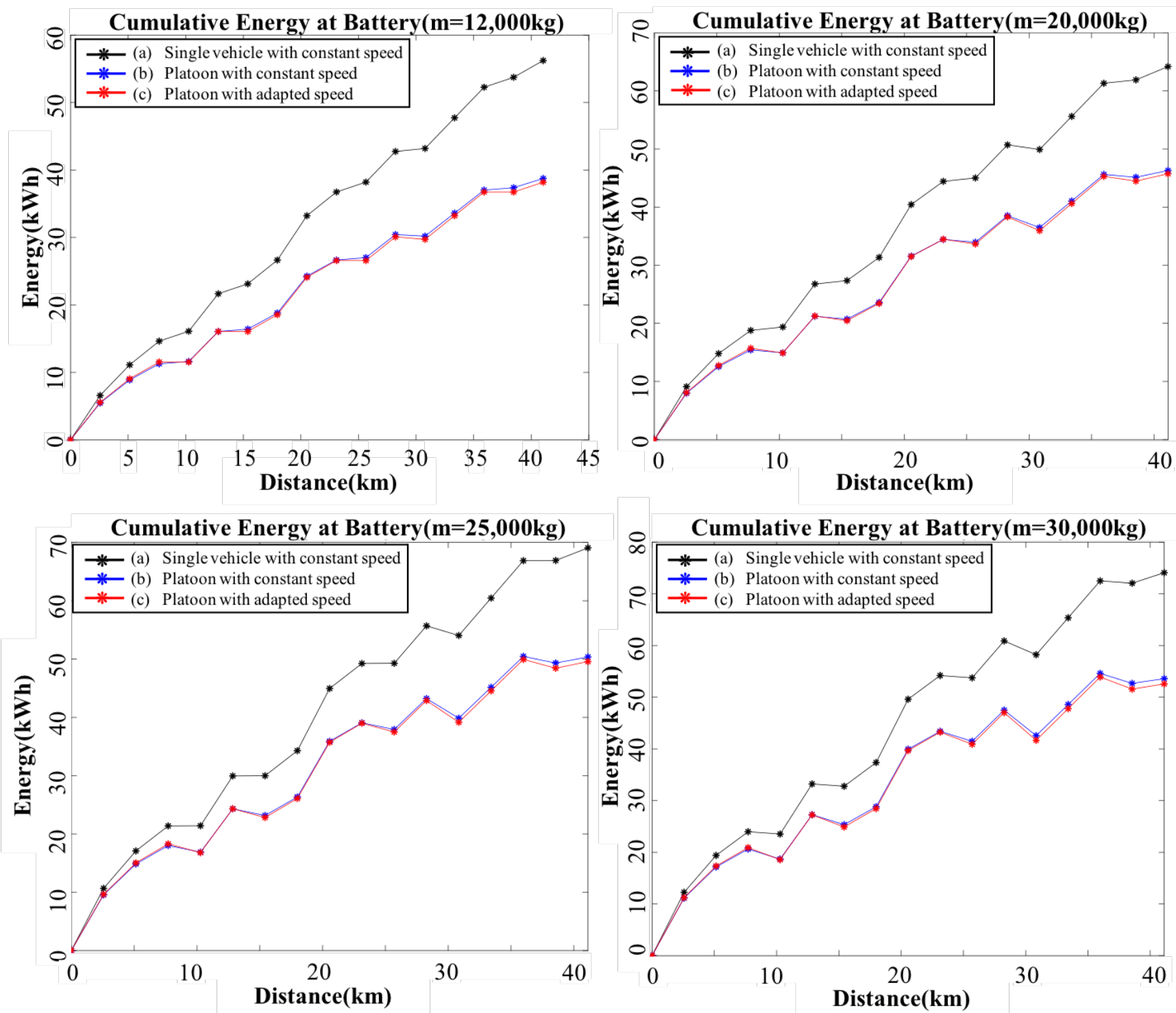

Figure 4. Energy consumption of four types of trucks with different $m / C_{d} A$.

For $12,000 \mathrm{~kg}$ trucks, the relative energy savings with regenerative braking in case (b) increased slightly compared to the case without regenerative braking, since it gained some energy through regenerative braking when declines are at $-3 \%$. However, relative energy savings in case (c) decreased a little compared to the case without regenerative braking. This is because we need to meet the constraint of travel time. The constant speed in case (c) with regenerative braking needs to be larger than without regenerative braking, which leads to more energy consumption. For 20,000 kg, 25,000 kg and 30,000 kg trucks, both the relative energy savings with regenerative braking in case (b) and (c) decreased since the average speeds decrease compared to the case without regenerative braking.

\subsection{Trade-off between Energy Savings and Travel Time}

For commercial HDV fleets, travel time is a critical factor that needs to be considered. Obviously, there is a trade-off between energy savings and travel time. After presenting the energy consumptions for four types of trucks in a platoon with the constraint of travel time in Sections 4.2 and 4.3, the constraint of time is now relaxed, and the energy savings are evaluated under different travel times. By changing the increase in travel time, the relative energy savings are simulated using four types of trucks for case (b) and (c) compared to the single truck with constant speed in case (a), and results are shown in Table 6. 
Table 6. Relative energy savings of case (b) and (c) with respect to the increase of travel time compared to the single truck case with constant speed in case (a).

\begin{tabular}{cccc}
\hline $\begin{array}{c}\text { Mass } \\
(\mathbf{k g})\end{array}$ & $\begin{array}{c}\text { Increase of Travel } \\
\text { Time }\end{array}$ & Case (b) & Energy Savings \\
& $3.9 \%$ & $31.5 \%$ & $34.5 \%$ \\
\multirow{2}{*}{12,000} & $9.5 \%$ & $33.6 \%$ & $39.6 \%$ \\
& $22.2 \%$ & $33.8 \%$ & $49.3 \%$ \\
20,000 & $3.9 \%$ & $27.9 \%$ & $30.4 \%$ \\
& $9.5 \%$ & $30.1 \%$ & $35.1 \%$ \\
25,000 & $22.2 \%$ & $33.8 \%$ & $42.9 \%$ \\
& $3.9 \%$ & $27.1 \%$ & $29.8 \%$ \\
& $9.5 \%$ & $28.7 \%$ & $34.0 \%$ \\
30,000 & $22.2 \%$ & $32.4 \%$ & $41.3 \%$ \\
& $3.9 \%$ & $27.7 \%$ & $30.5 \%$ \\
& $9.5 \%$ & $28.0 \%$ & $33.7 \%$ \\
\hline
\end{tabular}

From Table 6, the relative energy savings with speed adaption can be further increased to at most $49.3 \%$ with a travel time increase of $22.2 \%$. Lighter trucks gained more energy savings with less sacrifice of travel time than heavier trucks in a platoon.

\section{Conclusions}

This paper investigated the energy consumption of electric HDV platooning. We proposed an adaptive downhill coasting speed control method for highway situations to realize more energy savings for electric HDV platooning. By forming platoons using electric HDVs, our simulation results showed that electric platooning can provide at most $33.4 \%$ energy savings, which exceed those of ICE platooning (4.7-14.2\%) by a factor of two or more. In addition, by implementing our proposed adaptive downhill coasting speed profile, the energy savings of e-platooning can exceed by approximately $10 \%$ the case of constant speed. Moreover, we evaluated the trade-off between travel time and energy efficiency for the e-platooning with adaptive coasting speeds. It is shown that by increasing travel time by $22.2 \%$, the energy savings can be further increased to $49.3 \%$ in certain situations. It should be noted that a $49.3 \%$ reduction in battery energy corresponds to approximately a doubling of the vehicle range.

In this paper, to approximate the wide operating ranges of electric HDVs, the powertrain efficiency was approximated to be constant, which can be modified in future research. In addition, the change of road grades is an important factor in realizing downhill coasting speeds, so proper path planning might be a promising future research direction.

Author Contributions: Conceptualization, M.H. and P.B.; methodology, M.H. and P.B.; software, M.H.; validation, M.H., and P.B.; writing-original draft preparation, M.H.; writing-review and editing, M.H. and P.B. All authors have read and agreed to the published version of the manuscript.

Funding: This research received no external funding.

Conflicts of Interest: The authors declare no conflict of interest.

\section{References}

1. Daw, C.S.; Gao, Z.; Smith, D.E.; Laclair, T.J.; Pihl, J.A.; Edwards, K.D. Simulated fuel economy and emissions performance during city and interstate driving for a heavy-duty hybrid truck. SAE Int. J. Commer. Veh. 2013, 6, 161-182. [CrossRef]

2. Alam, A.A.; Gattami, A.; Johansson, K.H. An experimental study on the fuel reduction potential of heavy duty vehicle platooning. In Proceedings of the 13th international IEEE conference on intelligent transportation systems, Funchal, Portugal, 19-22 September 2010.

3. Liang, K.-Y.; Mårtensson, J.; Johansson, K.H. When is it fuel efficient for a heavy duty vehicle to catch up with a platoon? IFAC Proc. Vol. 2013, 46, 738-743. [CrossRef]

4. Bishop, R.; Bevly, D.; Switkes, J.; Park, L. Results of initial test and evaluation of a driver-assistive truck platooning prototype. In Proceedings of the 2014 IEEE Intelligent Vehicles Symposium Proceedings, Dearborn, MI, USA, 8-11 June 2014. 
5. Ellwanger, S.; Wohlfarth, E. Truck platooning application. In Proceedings of the 2017 IEEE Intelligent Vehicles Symposium (IV), Los Angeles, CA, USA, 11-14 June 2017.

6. Chan, E.; Gilhead, P.; Jelinek, P.; Krejci, P.; Robinson, T. Cooperative control of SARTRE automated platoon vehicles. In Proceedings of the 19th ITS World CongressERTICO-ITS EuropeEuropean CommissionITS AmericaITS Asia-Pacific, Vienna, Austria, 22-26 October 2012.

7. Alam, A. Fuel-Efficient Heavy-Duty Vehicle Platooning. Ph.D. Thesis, KTH Royal Institute of Technology, Stockholm, Sweden, 2014.

8. Sahlholm, P. Distributed Road Grade Estimation for Heavy Duty Vehicles. Ph.D. Thesis, KTH Royal Institute of Technology, Stockholm, Sweden, 2011.

9. Humphreys, H.; Bevly, D. Computational fluid dynamic analysis of a generic 2 truck platoon. SAE Tech. Pap. 2016, 1-8. [CrossRef]

10. Humphreys, H.L.; Batterson, J.; Bevly, D.; Schubert, R. An evaluation of the fuel economy benefits of a driver assistive truck platooning prototype using simulation. SAE Tech. Pap. 2016, 1-9.

11. McAuliffe, B.; Croken, M.; Ahmadi-Baloutaki, M.; Raeesi, A. Fuel-Economy Testing of a Three-Vehicle Truck Platooning System; National Research Council of Canada: Ottawa, ON, Canada, 2017.

12. Zhang, L.; Chen, F.; Ma, X.; Pan, X. Fuel Economy in Truck Platooning: A Literature Overview and Directions for Future Research. J. Adv. Transp. 2020, 2020, 2604012. [CrossRef]

13. Ohtsuka, T. A continuation/GMRES method for fast computation of nonlinear receding horizon control. Automatica 2004, 40, 563-574. [CrossRef]

14. Torabi, S. Fuel-Efficient Truck Platooning Using Speed Profile Optimization, Department of Mechanics and Maritime Sciences; Chalmers University of Technology: Gothenburg, Sweden, 2017.

15. He, C.R.; Ge, J.I.; Orosz, G. Fuel Efficient Connected Cruise Control for Heavy-Duty Trucks in Real Traffic. IEEE Trans. Control. Syst. Technol. 2020, 28, 2474-2481. [CrossRef]

16. Yoon, D.D.; Ayalew, B.; Ivanco, A.; Chen, Y. Predictive kinetic energy management for large electric vehicles using radar information. In Proceedings of the 2020 IEEE Conference on Control Technology and Applications, Montreal, QC, Canada, 24-26 August 2020; pp. 82-87.

17. Gazran, S.; Boukherroub, T.; Rönnqvist, M.; Paquet, M. Truck Platooning Transportation Planning: A Review with Emphasize on Operation Research Methods. Available online: https://www.researchgate.net/publication/349830460_Truck_platooning_ transportation_planning_A_review_with_emphasis_on_Operations_Research_methods (accessed on 5 October 2021).

18. USA Elevation Map. Available online: http://www.yellowmaps.com/maps/img/US/elevation/USA-elevation-map-242.jpg (accessed on 5 October 2021).

19. Hucho, W.; Sovran, G. Aerodynamics of road vehicles. Annu. Rev. Fluid Mech. 1993, 25, 485-537. [CrossRef]

20. Ehsani, M.; Gao, Y.; Longo, S.; Ebrahimi, K.M. Modern Electric, Hybrid Electric, and Fuel Cell Vehicles; CRC press: Boca Raton, FL, USA, 2018. 